\title{
Entry and Exit Mechanisms at the cis-Face of the Golgi Complex
}

\author{
Andrés Lorente-Rodríguez and Charles Barlowe \\ Department of Biochemistry, Dartmouth Medical School, Hanover, New Hampshire 03755 \\ Correspondence: charles.barlowe@dartmouth.edu
}

Vesicular transport of protein and lipid cargo from the endoplasmic reticulum (ER) to cisGolgi compartments depends on coat protein complexes, Rab GTPases, tethering factors, and membrane fusion catalysts. ER-derived vesicles deliver cargo to an ER-Golgi intermediate compartment (ERGIC) that then fuses with and/or matures into cis-Golgi compartments. The forward transport pathway to cis-Golgi compartments is balanced by a retrograde directed pathway that recycles transport machinery back to the ER. How trafficking through the ERGIC and cis-Golgi is coordinated to maintain organelle structure and function is poorly understood and highlights central questions regarding trafficking routes and organization of the early secretory pathway.

ewly synthesized secretory proteins and lipids are transported to early Golgi compartments in dissociative carrier vesicles that are formed from the ER (Palade 1975). Through the development of biochemical, genetic and morphological approaches, outlines for the molecular machinery that catalyze this transport step and the membrane structures that comprise the early secretory compartments have been developed (Rothman 1994; Schekman and Orci 1996). Initial images and studies suggested the early secretory pathway consisted of stable compartments with ER-derived carrier vesicles shuttling cargo to pre-Golgi and Golgi compartments. However, live cell and time-lapse imaging revealed highly dynamic structures with both secretory cargo and compartment residents detected in pleiomorphic intermediates (Presley et al. 1997; Scales et al. 1997; Shima et al. 1999; Ben-Tekaya et al. 2005). Several lines of evidence now indicate that active bidirectional transport cycles between the ER, ERGIC, and cis-Golgi compartments are balanced to maintain compartment structure and function (Lee et al. 2004). The coat protein complex II (COPII) produces vesicles for forward transport from the ER whereas the coat protein complex I (COPI) buds vesicles for retrograde transport from cis-Golgi compartments to the ER (Fig. 1). Indeed inhibition of either the forward or retrograde pathways results in a rapid loss of normal ER and Golgi organization (Lippincott-Schwartz et al. 1989; Rambourg et al. 1994; Morin-Ganet et al. 2000; Ward et al. 2001). Yet, under steady-state conditions, the cis-Golgi compartments retain a characteristic composition and structure as secretory cargo passes through. These observations indicate

Editors: Graham Warren and James Rothman

Additional Perspectives on The Golgi available at www.cshperspectives.org

Copyright (C) 2011 Cold Spring Harbor Laboratory Press; all rights reserved; doi: 10.1101/cshperspect.a005207 Cite this article as Cold Spring Harb Perspect Biol 2011;3:a005207 


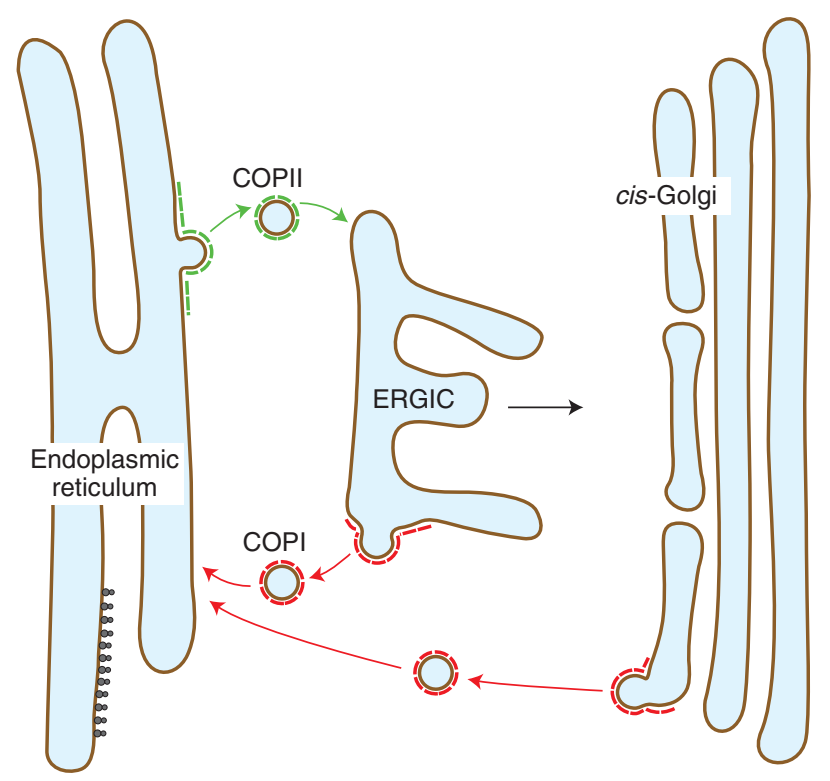

Figure 1. Trafficking in the early secretory pathway. A simplified model depicting bidirectional transport routes between the endoplasmic reticulum (ER), the ER-Golgi intermediate compartment (ERGIC), and cis-Golgi cisternae. COPII vesicles bud from the ER and transport cargo in an anterograde direction for tethering and fusion with the ERGIC. The ERGIC then matures into the cis-Golgi compartment and/or fuses with the cis-Golgi. COPI vesicles bud from the ERGIC and cis-Golgi compartments in a retrograde transport pathway back to the ER to recycle transport machinery.

that the structure and function of early secretory compartments are maintained at dynamic equilibrium through coordination of multiple pathways.

Although much of the molecular machinery required for transport to and from cis-Golgi membranes has been identified, the current challenges are to understand the sequence of molecular events that underlie the observed structural organization. Moreover, the mechanisms that govern entry and exit rates to-from cis-Golgi membranes to maintain organization at dynamic equilibrium are poorly understood. In this perspective the known key machinery required for anterograde transport to, and retrograde exit from, cis-Golgi compartments will be described, and then current models for how compartmental structure is maintained while protein and lipid cargo fluxes through will be examined. This review will survey core machinery and principles from several experimental models although it is becoming clear that different species and different cell types within a species may employ variations on a basic theme depending on cellular function. For example, in many animal cells secretory cargo passes through a well-characterized ERGolgi intermediate compartment (ERGIC) en route to cis-Golgi cisternae. The ERGIC may be necessary to span the distance between ER export sites and the Golgi stack; however, the relationship of this intermediate compartment to the cis-Golgi remains unclear (AppenzellerHerzog and Hauri 2006). In other species including yeast, Golgi organization appears less coherent and may be considered more simply as a series of intermediate compartments. Here COPII vesicles are thought to fuse directly with the cis-Golgi or this first intermediate compartment (Fig. 2) (Mogelsvang et al. 2003). Despite these quite varied structural organizations, there is a remarkable level of conservation in molecular machinery. After consideration of this conserved molecular machinery, we will return to organizational issues in transport to and from the cis-Golgi compartment. 

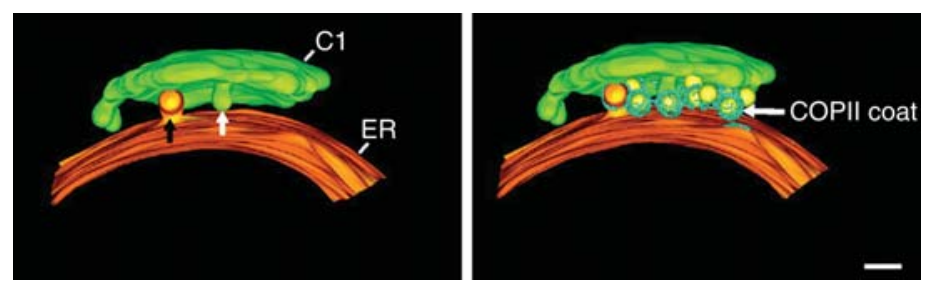

Figure 2. ER/Golgi organization in the yeast P. pastoris. Three-dimensional models reconstructed from electron microscope tomography showing proximity of ER and cis-most cisterna of the Golgi complex. Putative COPII vesicles are detected in a narrow region between the ER and cis-Golgi. For further information, see Mogelsvang et al. (2003). Bar $=100 \mathrm{~nm}$. (Reprinted, with permission, from Mogelsvang et al. 2003 [American Society for Cell Biology].)

\section{VESICLE TETHERING MACHINERY AT CIS-GOLGI COMPARTMENTS}

Through a combination of experimental approaches, investigators have identified distinct sub-stages in the pathway of protein transport from the ER to the Golgi complex. In general, transport intermediates bud from the ER and are guided to acceptor membranes through a series of targeting events that ultimately lead to pairing of vesicle and acceptor membrane SNARE (soluble NSF-attachment protein receptor) proteins to drive bilayer fusion. The initial targeting events can include longer distance movements guided by microtubule directed motors followed by Rab GTPase-dependent tethering through filamentous type proteins that contain extended $\alpha$-helical coiled-coil domains. Once transport intermediates are tethered to appropriate acceptor membranes, or perhaps concomitant with tethering, the multisubunit transport protein particle I (TRAPP I) binds vesicles in closer apposition to membranes for assembly of the SNAREprotein dependent fusion machinery. Cell-free approaches have been devised to measure distinct stages in vesicle tethering and fusion with Golgi compartments through co-sedimentation and compartment mixing assays (Beckers et al. 1987; Baker et al. 1988; Cao et al. 1998; Barrowman et al. 2000). In vivo molecular genetic approaches combined with microscopy have been used to determine requirements for transport and organization of early secretory compartments as well as to monitor the directed movement of transport intermediates (Novick et al. 1980; Pepperkok et al. 1993; Presley et al. 1997; Scales et al. 1997; Puthenveedu and Linstedt 2004). Through these approaches, an extensive set of molecular requirements and protein-protein interactions have now been described. Interestingly, there is increasing evidence for overlapping associations between the protein components involved in the vesicle budding, tethering, and fusion stages that probably reflect a network of interactions necessary to ensure high fusion specificity at cis-Golgi compartments. The Rab GTPase cycle and its regulators appear to play a central role in providing directionality to the core transport machinery. It is this machinery that will be examined in more detail in the following sections, starting with the vesicle tethering stage.

\section{The Extended Coiled-Coil Domain Tethers}

The p115 protein in animal cells and orthologous Usol protein in yeast are required for traffic to the Golgi in vivo (Nakajima et al. 1991; Puthenveedu and Linstedt 2001; Puthenveedu and Linstedt 2004) and in cell free assays that measure vesicle transport from the ER to the Golgi (Barlowe 1997; Alvarez et al. 1999; Allan et al. 2000). Purified p115 in active form is a myosin-shaped parallel homodimer that consists of an amino-terminal globular domain, an extended flexible rod composed of a series of $\alpha$-helical coiled-coil domains, followed by a 20 amino acid stretch of acidic residues at the carboxyl terminus. Structural studies suggest that p115 spans a distance of $60 \mathrm{~nm}$ from 
head to tail whereas Usol has an even longer coiled-coil segment for a total length of approximately $180 \mathrm{~nm}$ (Sapperstein et al. 1995; Yamakawa et al. 1996). Functional analyses show that these extended proteins can tether COPII and COPI vesicles to Golgi membranes in a stage that precedes membrane fusion (Cao et al. 1998; Sönnichsen et al. 1998). The p115/ Uso1-dependent vesicle-tethering event is thought to restrict vesicle diffusion and favor site-specific fusion with the target membrane. Indeed evidence indicates that p115 attaches to forming COPII transport vesicles at ER export sites to increase the efficiency of tethering to subsequent acceptor compartments (Allan et al. 2000; Kondylis and Rabouille 2003).

What are the molecular contacts that connect p115/Uso1 to both ER-derived transport vesicles and to Golgi acceptor membranes? The Rab GTPase Rab1 in animal cells and Ypt1 in yeast perform a critical role in p115/ Uso1 targeting, possibly acting on both donor and acceptor compartments. Several studies have shown a role for this small GTPase in recruiting $\mathrm{p} 115 /$ Usol to membranes (Cao et al. 1998; Allan et al. 2000). Precisely how Rab1 controls p115 membrane association is not clear although studies have indicated a direct association through a region in the globular head domain of p115 (An et al. 2009) and through a second site in the first coiled-coil domain of the extended $\alpha$-helical rod (Beard et al. 2005). Interestingly, the carboxy-terminal tail of p115 may fold back to auto-inhibit the Rab1 binding site in the first coiled-coil domain (Beard et al. 2005). In addition to these Rab1 binding sites, the domain architecture of p115 appears to provide multiple interaction surfaces to regulate membrane tethering and to coordinate with other components of the tethering and fusion machinery. For example, p115 is also detected in association with the GRASP65GM130 tethering complex (Moyer et al. 2001) and with specific Golgi SNARE proteins (Allan et al. 2000; Shorter et al. 2002) that function in fusion of ER-derived vesicles with Golgi acceptor membranes. As we will see following further analyses of other ER/Golgi tethering and fusion machinery components, multiple modes of binding will be a reoccurring theme and suggest that a sequential series of associations may transfer targeted intermediates along a directed pathway to site-specific membrane fusion.

Similar to p115, GRASP65, and GM130 also contain extended $\alpha$-helical coiled-coil domains as do a larger class of "golgins" that are reviewed in Munro (2011) and are known to localize across Golgi compartments (Barr 1999; Barr and Short 2003). The GRASP65-GM130 complex is anchored to Golgi membranes through amino-terminal myristoylation of GRASP65 and association with Rab1 (Barr et al. 1998; Allan et al. 2000). In vitro assays show a role for the GRASP65-GM130 complex in consumption of COPII vesicles at cis-Golgi compartments (Moyer et al. 2001; Alvarez et al. 2001; Shorter et al. 2002). However, siRNA experiments indicate that knockdown of GRASP65 and GM130 do not block transport through the secretory pathway but do influence normal structural organization of the Golgi stack (Tang et al. 2010). In contrast p115 knockdown blocks transport through the early secretory pathway (Puthenveedu and Linstedt 2001; Kondylis et al. 2005; Puthenveedu et al. 2006).

In yeast cells, there are no clear orthologs of GM130 but there is a GRASP65 homolog, known as Grh1, in addition to other golgin-like proteins including Bug1, Coy1, and Rud3. Bug1 binds to Grh1 and this complex is anchored to membranes through an acetylated amino-terminal region in Grh1. In vitro assays show that Grh1 and Bug1 are required for optimal Uso1-dependent tethering (Behnia et al. 2007). Interestingly, Grh1 also interacts with the Sec23/24 subunit of the COPII coat and is detected at ER export sites as well as on COPII vesicles (Behnia et al. 2007; Levi et al. 2010). As suggested for p115, COPII vesicles may be programmed for tethering with the ERGIC or cis-Golgi compartments by recruitment of Ghr1 to forming vesicles. As in animal cells, Grh1 and Bug1 are not required for secretory pathway function and display only modest trafficking defects when absent. However, the genes encoding Grh1, Bug1, Coyl, and Rud3 do display an array of synthetic negative 
relationships with other genes involved in transport to the Golgi, most notably with the essential genes YPT1 and USO1 (VanRheenen et al. 1999; Gillingham et al. 2002; Gillingham et al. 2004; Behnia et al. 2007).

These collective findings suggest that, with the exception of p115/Uso1, there is a redundant network of coiled-coil golgins at the ERGIC and cis-Golgi such that removal of a single member permits transport to the Golgi, presumably through ample tethering activity provided by other golgin members. It does, however, remain possible that p115/ Uso 1 could act with distinct golgins in multiple SNARE-dependent fusion pathways and inhibition of one route could be compensated by increased activity though a parallel or secondary pathway.

\section{The Multisubunit Tethering Complex TRAPP |}

Although coiled-coil domain tethers can potentially span long distances to connect vesicles with acceptor membranes, the transport protein particle I (TRAPP I) complex is also required for COPII-dependent transport to cis-Golgi compartments in vivo and in vitro (Rossi et al. 1995; Sacher et al. 1998). Moreover, in vitro assays show that TRAPP I can physically link COPII vesicles to Golgi acceptor membranes (Sacher et al. 2001). Structural studies show that TRAPP I is a $170 \mathrm{kDa}$ particle consisting of six subunits (Bet3, Bet5, Trs20, Trs23, Trs31, Trs33) with a stoichiometry of two Bet3 subunits per complex and one copy each of the other subunits. Combined EM and crystallographic analyses of purified TRAPP I, and of specific TRAPP I sub-complexes, reveal a bi-lobed arrangement with dimensions of approximately $18 \times 6 \times 5 \mathrm{~nm}$. Notably, the two Bet 3 subunits are positioned on opposite ends of the bi-lobed TRAPP I structure (Kim et al. 2006). In animal cells, TRAPP I appears to act in homotypic fusion of COPII vesicles and may employ this symmetrically positioned Bet3 for such a purpose (Yu et al. 2006; Cai et al. 2007a). Recent studies show that the Bet3 subunit of TRAPP I binds directly to the Sec23 subunit of COPII and this interaction is important for tethering COPII vesicles in yeast and in animal cells (Cai et al. 2007b). The membrane orientation of TRAPP I in homotypic and heterotypic tethering remains to be determined although two modes of binding have been proposed. For heterotypic membrane fusion TRAPP I is thought to lie flat on the ERGIC or cis-Golgi membrane surface and present conserved residues facing the cytoplasm to coordinate vesicle tethering with SNARE dependent fusion (Kim et al. 2006). In homotypic membrane fusion events, TRAPP I has been proposed to bind end-on such that Bet3 would bind Sec 23 on apposed vesicles to tether COPII vesicles (Cai et al. 2007a).

Importantly, the TRAPP I complex also functions as a guanine nucleotide exchange factor (GEF) for Yptl and this activity is thought to generate activated Ypt1 GTPase on the surface of Golgi acceptor membranes (Wang et al. 2000; Jones et al. 2000). The GEF activity minimally depends on a sub-assembly of TRAPP I subunits consisting of Bet3, Bet5, Trs23, and Trs31. Recent determination of the crystal structure of this TRAPP I subassembly in complex with Ypt1 indicates that Bet3, Bet5, and Trs23 all contact Ypt1 directly and catalyze nucleotide exchange by stabilizing an open form of the GTPase (Cai et al. 2008). Given that p115/ Uso1 and potentially other golgins are Rab effectors that bind to the activated GTP form of Rab1/Ypt1, we are beginning to see how these TRAPP I and p115/Uso1 activities could be coordinated through Rab GTPases in membrane tethering. One possibility is that TRAPP I locally activates Rab proteins to recruit p115/Usol and possibly other golgins to a region on the acceptor membrane surface. Vesicles initially tethered by p115/Uso1 could then be directed into a more membrane proximal position through interactions between vesicle bound Sec23 and TRAPP I. Finally, TRAPP I could also connect tethering with SNARE-dependent membrane fusion through direct interactions with specific SNARE proteins. Although no physical connections between TRAPP I and the ER/Golgi SNARE proteins have been detected, it is noteworthy that the mammalian ortholog of Trs20, known 
as sedlin, contains an amino-terminal domain structurally related to amino-terminal regulatory domains reported in SNARE proteins (Jang et al. 2002; Kim et al. 2006). In this manner, TRAPP I could serve as a platform on the Golgi surface to first locally capture vesicles on a coiled-coil tether, then bind vesicles in closer proximity to the acceptor membrane and finally promote trans-association of vesicle and acceptor membrane SNARE proteins. Although additional work will be required to determine the molecular contacts and arrangement(s) of TRAPP I in tethering, this multisubunit tethering complex appears to be a central hub in coordinating fusion events at the cis-Golgi (Fig. 3).

\section{MEMBRANE FUSION AT THE ERGIC AND CIS-GOLGI COMPARTMENTS}

Fusion of tethered membranes at the ERGIC and cis-Golgi compartments depends on SNARE proteins, a set of tail-anchored integral membrane proteins that contain a conserved membrane proximal heptad repeat sequence known as the SNARE motif (Rothman 1994). Intracellular membrane fusion in the exocytic and endocytic trafficking pathways is catalyzed through a conserved mechanism in which SNARE motifs on the surface of donor and acceptor membranes are assembled into stable four-helix bundles. The close apposition of membranes following assembly of SNARE complexes in trans is thought to strain membranes in a manner that induces controlled bilayer fusion (Weber et al. 1998). The center or "zero layer" of a cognate four-helix bundle consists of ionic residues such that three of the SNARE proteins contribute a glutamine residue, and are termed Q-SNAREs, whereas the fourth helix contains an arginine residue, and is termed an R-SNARE (Fasshauer et al. 1998). Further classification of Q-SNARE proteins based on sequence conservation places each member into the $\mathrm{Qa}, \mathrm{Qb}$, or Qc subfamily (Bock et al. 2001). Current models indicate that fusogenic SNARE complexes are formed

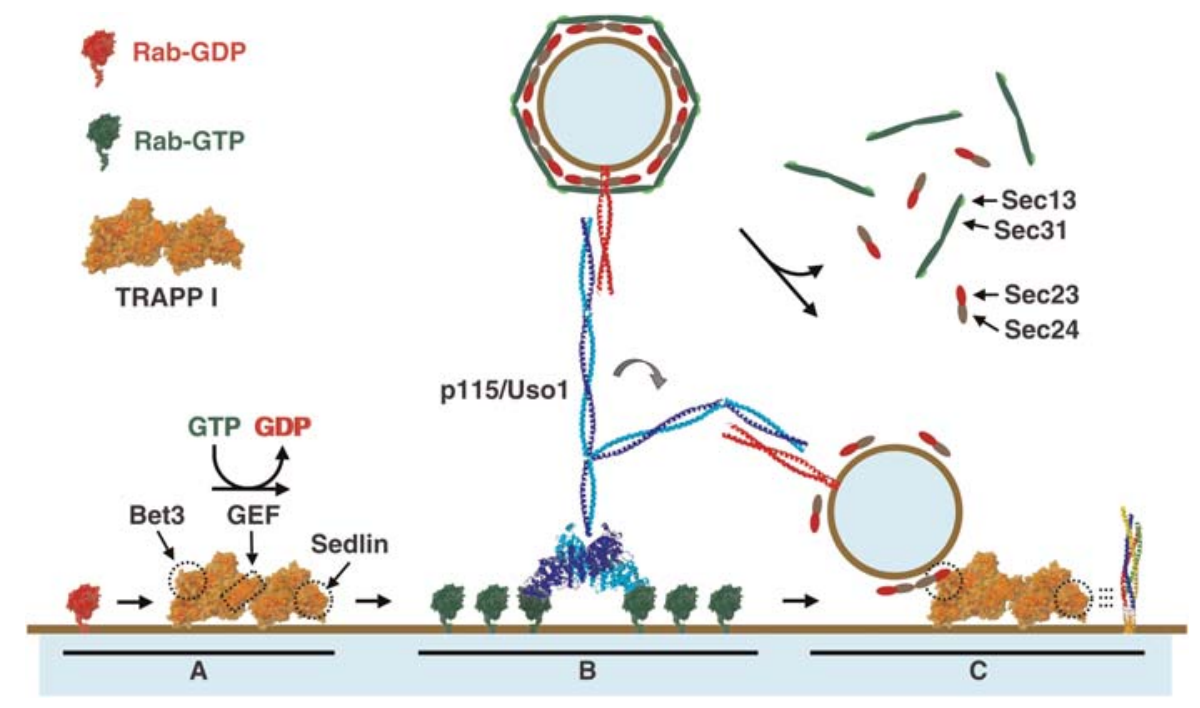

Figure 3. Model of COPII vesicle tethering. (A) TRAPP I catalyzes nucleotide exchange on membrane bound Rab1/Yptl-GDP (red), generating a tethering/fusion domain at the acceptor membrane enriched in Rabl/ Ypt1-GTP (green), tethering factors and SNARE proteins $(B-C)$. The guanine nucleotide exchange factor (GEF), Bet3 and Sedlin/Trs20 regions of TRAPP I are indicated. (B) Rab1/Ypt1-GTP recruits p115/Uso1 as well as other coiled-coil golgins, which may interact with coiled-coil tethering factors present on the vesicle. (C) p115/Uso1 may bring the vesicle closer to the membrane and promote TRAPP I vesicle tethering mediated by interactions between Bet 3 and Sec23. Sedlin/Trs20 may interact with SNARE proteins (far right). ( $A-C$ ) The illustrations of factors involved in tethering are based on published structures available in PDB. 
in trans by assembly of three Q-SNAREs (Qa, $\mathrm{Qb}$, and Qc) and one R-SNARE, through a zippering process from the distal amino-terminal side of SNARE motifs toward the membrane (Sudhof and Rothman 2009). In the case of ER to Golgi SNARE-dependent fusion, several lines of evidence indicate that Sed5 (Qa), Bos1 (Qb), Bet1 (Qc), and Sec22 (R-SNARE) catalyze fusion between COPII vesicles and Golgi acceptor membranes in yeast (Lee et al. 2004). Indeed, this minimal set is capable of driving fusion in reconstituted proteoliposomes (Parlati et al. 2000). In animal cells, an ortholgous set of SNAREs known as syntaxin 5 (Qa), membrin $(\mathrm{Qb})$, rbet $(\mathrm{Qc})$, and sec22b (R-SNARE) appear to drive fusion between COPII vesicles and early Golgi compartments (Hay et al. 1997; Hay et al. 1998; Rowe et al. 1998; Xu et al. 2000). Experimental evidence also suggests that this set can drive homotypic as well as heterotypic membrane fusion events (Rowe et al. 1998; Xu and Hay 2004). A more complete review of the SNARE protein machinery that operates within the Golgi stack can be found in Malsam and Söllner (2011).

In addition to this core SNARE protein fusion machinery, SNARE regulatory proteins are required to control fusion specificity and to recycle postfusion cis-SNARE complexes. Members of the Sec1/Munc18-1 (SM) family of proteins are required for each SNAREdependent fusion event. The SM protein Sly1 is required for fusion of COPII vesicles with Golgi acceptor membranes in yeast and in animal cells (Ossig et al. 1991; Dasher et al. 1996; Cao et al. 1998). The SLY1 gene in yeast was originally identified as a suppressor of loss of YPT1 function when the gain of function SLY1-20 allele was isolated in a selection screen for mutations that would permit growth in the absence of the normally essential YPT1 gene. Structural and biochemical studies on Sly1 and other SM proteins indicate that these factors undergo conformational switches when bound to the amino-terminal regulatory domain of Sed5/Qa-SNARE family members in addition to other sites within a forming trans-SNARE complex (Shen et al. 2007; Dulubova et al. 2007; Furgason et al. 2009). Sly1 has also been shown to impart specificity in assembly of cognate SNARE complexes (Sed5, Bos1, Bet1, Sec22) compared to nonphysiological SNARE complex associations (Peng and Gallwitz 2002). Current models posit that SM proteins bind Qa-SNARE proteins through multiple modes to regulate assembly and ultimately act as a clamp to stabilize cognate transSNARE complexes (Sudhof and Rothman 2009; Furgason et al. 2009). Precisely how Sly1 and other SM proteins catalyze SNARE complex assembly and what upstream signals may regulate their activity remain active areas of study.

After SNARE-mediated membrane fusion events are completed, stable four-helix bundle cis-SNARE complexes reside in a single membrane bilayer. To reuse the assembled Sed5Bos1-Bet1-Sec22 complex proteins for additional rounds of membrane fusion, NSF/Sec18 in concert with SNAP/Sec17 catalyzes SNARE complex disassembly. NSF/Sec18 belongs to the AAA-family of ATPase chaperones and harnesses the energy of ATP hydrolysis to separate thermodynamically stable cis-SNARE complexes. SNAP/Sec17 binds to SNARE proteins and couples NSF/Sec18 ATP-dependent activity to dissociate cis-SNARE complexes (Sudhof and Rothman 2009). How this SNARE disassembly cycle is regulated and coordinated with other budding and fusion events at the ERGIC and cisGolgi compartments is not well understood.

Genetic experiments in yeast have been informative in assessing relationships between cis-Golgi tethering factors, Ypt1, Sly1, and the ER/Golgi SNARE proteins. A particularly insightful gain of function mutation in Sly1, mentioned above as SLY1-20, restores viability to yeast cells in the absence of Ypt1 (Dasher et al. 1991). Moreover, the SLY1-20 allele can also suppress deficiencies in USO1 (Sapperstein et al. 1996). In addition to SLY1-20, the screen for dosage suppressors of loss of Ypt1 also uncovered additional multicopy suppressor genes that encode the ER-Golgi Qc-SNARE Bet1 and the R-SNARE Sec22 (Dascher et al. 1991). These findings indicate that when the tethering step is diminished, elevation of SNARE activity, either by activation of the SM protein Sly1, or by increasing the 
concentration of SNARE protein complexes, can overcome tethering deficits. Over-expression of Ypt1 also restores growth of the uso1 $\Delta$ and bet5-1 mutant strains suggesting these activities can be bypassed when Ypt1 levels are increased (Sapperstein et al. 1996; Jiang et al. 1998). Finally, when multiple cis-Golgi tethering factors are deleted, such as in a uso $1 \Delta$ grh1 $\Delta$ double mutant strain, the SLY1-20 allele no longer supports growth (Behnia et al. 2007). These collective findings are consistent with an order of events in which a redundant network of coiled-coil tethering proteins act upstream of TRAPP I and Ypt1 followed by the SM protein Sly1 and lastly the SNARE protein fusion catalysts.

Toward a Model for Concerted Vesicle Tethering and Membrane Fusion

Based on the current biochemical, genetic and cell biological evidence, an outline for the continuum of events leading from vesicle tethering to bilayer fusion at the ERGIC and cisGolgi can be envisaged (Figs. 3 and 4). TRAPP I could serve as an organizing hub using its GEF activity to generate a local concentration of Rab1/Ypt1-GTP near SNARE membrane fusion machinery. Activated Rab1/Ypt1-GTP would then bind p115/Usolp and potentially other coiled-coil tethering factors to this localized region. Interactions between extended coiled-coil domain proteins on COPII vesicles and acceptor membranes, through parallel or antiparallel orientations, could initially tether vesicles to incipient membrane fusion sites. Partially coated tethered vesicles would then be spatially restricted in a manner that would favor associations with TRAPP I and position vesicle and acceptor SNARE proteins in close proximity. The TRAPP I bound vesicle could transmit signals to downstream fusion machinery in preparation for bilayer fusion, perhaps by a further increase in Rab1/Ypt1-GTP levels or by direct contact with fusion factors. The result of this signal would be to prime cis-SNARE complexes and/or generate SM-SNAREprotein conformation changes that promote assembly of fusogenic trans-SNARE complexes. Zippering up of helical bundles into transSNARE complexes would presumably then lead to rapid hemi-fusion followed by bilayer fusion and content mixing (Sudhof and Rothman 2009). Obviously several mechanistic

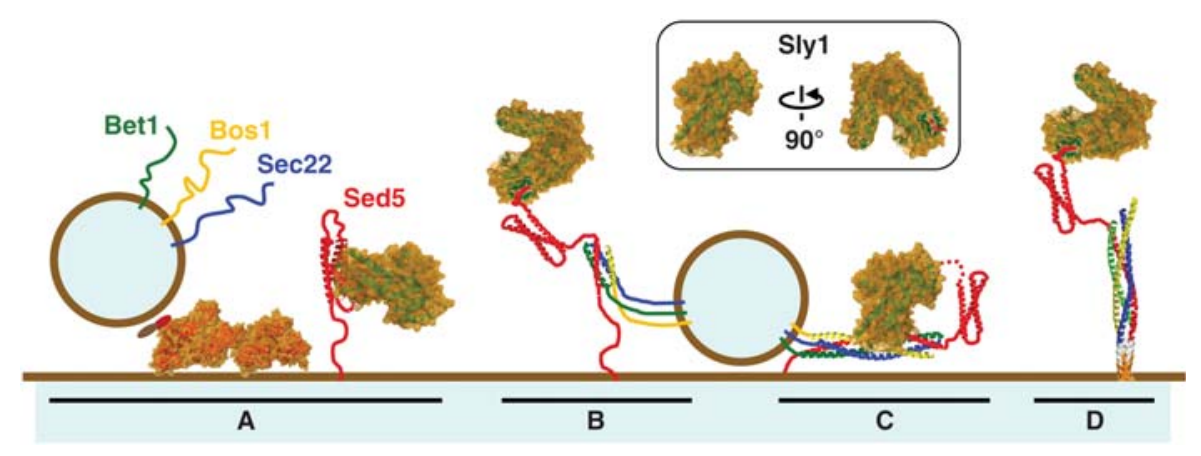

Figure 4. Model of COPII vesicle fusion. (A) Uncoated vesicles display vesicle SNARE proteins primed for fusion. At the acceptor membrane, Sly1 may bind the closed conformation of Sed5 preventing its association with noncognate SNAREs. A possible simultaneous binding to the Sed5 amino-terminal peptide is not shown. (B) Sly1 bound to the Sed5 amino-terminal peptide allows Sed5 to interact with vesicle SNAREs. $(B-C)$ SNARE proteins assemble into parallel four- $\alpha$-helical trans-SNARE bundles. Sly1 is proposed to promote formation of cognate trans-SNARE complexes. $(C)$ As for other SM proteins, Sly1 may also bind the trans-SNARE bundle to stabilize assembled complexes. Potential simultaneous binding to the Sed5 amino-terminal peptide is depicted as a dotted line. $(D)$ Membrane fusion is catalyzed by zippering into a four-helix bundle. $(A-D)$ The cartoons of factors involved in fusion are based on published structures available in PDB. Inset shows two views of Sly1; region in red indicates site of insertion of the Sed5 amino-terminal peptide. 
issues need to be resolved for a full understanding of this pathway. For example, what anchors TRAPP I to membranes and how might a membrane domain rich in TRAPP I, Rab1/Ypt1, and SNARE proteins be produced? Once tethering interactions are formed what regulates their dissociation? Which SNARE proteins operate on which compartments or can specific SNARE proteins act on either compartment? Finally, is tethering and fusion a constitutive process under "substrate control" or are there sensor mechanisms in place to regulate transport rates to the cis-Golgi? There are clearly multiple nodes in this transport pathway in which regulation could be imposed. We will return to this regulatory issue after review of the COPIdependent retrograde pathway from cis-Golgi compartments and then consider how these pathways are coordinated to maintain cis-Golgi organization.

\section{COP-I-DEPENDENT EXPORT FROM CIS-GOLGI COMPARTMENTS}

The structure and function of cis-Golgi compartments is maintained at dynamic equilibrium in part through the activity of a robust COPI-dependent retrograde pathway that operates to recycle vesicle proteins and lipids for use in multiple rounds of transport between the ER and Golgi. In essence, all of the protein constituents on COPII vesicles that are required for cargo selection, vesicle tethering and SNARE-dependent fusion are recycled back to the ER or to an intermediate compartment in COPI vesicles. For retrograde transport to the $\mathrm{ER}$, the Dsll tethering complex functions in this pathway to target COPI vesicles for SNAREdependent fusion at the ER (Yu and Hughson 2010). COPI, or coatomer-derived vesicles were first molecularly characterized through the use of in vitro assays measuring intra-Golgi transport (Malhotra et al. 1989; Waters et al. 1991). Assembly of COPI coats at the cis-Golgi depends on the small GTPase Arf1 (Serafini et al. 1991; Spang and Schekman 1998) and membrane bound Arf1-GTP recruits the heptameric coatomer complex en bloc to Golgi membranes to form COPI vesicles (Ostermann et al.
1993). Arf1-GTP is generated on the surface of cis-Golgi membranes by Arf1-specific guanine nucleotide exchange factors (GEFs) belonging to the conserved GBF/Gea family (Bui et al. 2009). The Arf1 GEFs are large multidomain proteins in peripheral association with the ERGIC and Golgi membranes and perform a critical role in controlling the localized assembly of COPI coated vesicles (Zhao et al. 2002; García-Mata et al. 2003). On the other side of the Arf1 GTPase cycle, Arf1 specific GAPs that belong to the Arf-GAP1/Gcs1 family appear to function in cargo selection as well as in coat disassembly in a manner that may sense membrane curvature (Spang et al. 2010). Through a powerful combination of structural biology and biochemical assays, our understanding on the molecular mechanisms in COPI coat assembly are advanced and are described in this series of perspectives (Wieland 2011). For our consideration of exit rates from cis-Golgi compartments it is important to appreciate that core subunits of the heptameric coatomer complex function as cargo adaptors for retrograde cargo through recognition of cytoplasmically exposed sorting signals that include the carboxy-terminal di-lysine based signals (Letouneur et al. 1994; Pavel et al. 1998). Therefore COPI plays an important sorting role in selecting and retaining retrograde cargo while biosynthetic secretory proteins advance.

What are the mechanisms that govern rates of COPI-dependent retrograde transport from the ERGIC and cis-Golgi compartments? Clear answers are not known but experimental evidence suggests at least two obvious modes of regulation. First, the relative abundance of retrograde directed cargo present in the ERGIC or cis-Golgi membranes could drive budding rates by mass action. The p24 family of type I transmembrane proteins are particularly abundant in COPI as well as COPII vesicles (Stamnes et al. 1995; Belden and Barlowe 1996) and present potent di-lysine sorting signals on the cytosolic surface of membranes that are known to stimulate reconstituted COPI vesicle formation from synthetic liposomes (Bremser et al. 1999). The presence of $\mathrm{p} 24$ proteins in cis-Golgi membranes has also been shown to facilitate 
COPI budding in cell-free assays that recapitulate retrograde directed transport (AguileraRomero et al. 2008). Moreover, the presence of specific retrograde SNARE proteins could serve as primers for COPI vesicle assembly (Springer et al. 1999) and are observed to recruit COPI components in an Arf-GAP dependent manner (Rein et al. 2002). Therefore, the overall concentration of COPI sorting signals in ERGIC and cis-Golgi membranes could serve to regulate COPI budding rates.

A second potential site of regulation centers on the Arf1-GEF activities catalyzed by the multidomain GBF1/Gea proteins. Initiation of COPI assembly on cis-Golgi membranes is temporally and spatially controlled by Geal (Guanine nucleotide Exchange on ARF) in yeast cells and the orthologous GBF1 (Golgi-associated BFA-resistant) in animal cells. These Arf1-GEFs are targets of the fungal metabolite brefeldin A (BFA), which triggers rapid Golgi disassembly after brief treatment (LippincottSchwartz et al. 1989; Peyroche et al. 1996; Niu et al. 2005). BFA traps Arf1-GEFs in a dead-end complex with Arf1-GDP and effectively blocks generation of Arf1-GTP (Peyroche et al. 1999). The rapid loss of cis-Golgi structure and function following inhibition of Arfl-GEF activity highlights a central role played by the COPIdependent retrograde pathway in Golgi organization. Moreover, the multidomain architecture of the Arf1 GEFs is potentially well suited to serve in regulation of COPI assembly. The ARFGEF activity of GBF1 resides in a conserved central 200 amino acid residue "Sec7 domain" but this 1855 amino acid protein also contains at least five other identifiable domains (Mouratou et al. 2005). The amino-terminal DCB (dimerization and cyclophilin binding) domain and HUS (homology upstream of Sec7) are thought to play roles in GBF1 localization. Additional conserved carboxy-terminal domains could potentially serve to sense or receive signals reporting on lipid and/or protein status to control exit rates. A recent study documents that phosphorylation and membrane dissociation of GBF1 occurs as cells enter mitosis (Morohashi et al. 2010) again pointing to an important regulatory role for ARF GEF activity. Although much remains to be understood regarding the Arf1-GEF proteins, a mechanistic dissection of their activities appears to represent an exciting avenue of study.

\section{ORGANIZATIONAL ISSUES AND FUTURE QUESTIONS}

Several lines of evidence indicate that ERderived vesicles deliver nascent cargo to cisGolgi compartments in a transport process that depends on tethering factors, small GTPases and SNARE proteins. But how are the early secretory compartments organized and where precisely do ER-derived vesicles first fuse? The potential COPII vesicle fusion targets are with one another (homotypic fusion), with an existing ERGIC, with retrograde directed COPI vesicles or directly with cis-Golgi membranes. High resolution analyses of ER/Golgi structures in yeast using electron microscopy (EM) document the presence of vesicles adjacent to cis-Golgi compartments (Mogelsvang et al. 2003). These observations together with in vitro studies in yeast suggest that ER-derived vesicles undergo heterotypic fusion with cisGolgi membranes. However, this cis-Golgi compartment in yeast could alternatively be considered a first intermediate compartment, or ERGIC, in a series that makes up the yeast Golgi complex. Although homotypic fusion of COPII vesicles may occur in yeast, there is currently no evidence to support this event as a major transport route.

EM analyses of the ER/Golgi interface in animal cells document the ERGIC as a collection of vesicular-tubular elements positioned adjacent to ER-exit sites (Bannykh et al. 1996). Experiments in animal cells also suggest that homotypic COPII vesicle fusion can occur in vitro (Xu and Hay 2004) although it remains to be determined if this is a major transport route in vivo. However, even if homotypic fusion of COPII vesicles occurs, there must be a heterotypic fusion event that mixes the content of COPII vesicles with the ERGIC because the composition of COPII vesicles and the ERGIC appear to be distinct. Heterotypic fusion could occur between COPII vesicles 
and the ERGIC or between COPII vesicles and COPI vesicles to generate the ERGIC. Indeed, both of these fusion events may need to occur. Notably, the composition of the ERGIC is similar to cis-Golgi membranes with appropriate tethering factors, Rab1 GTPase and ER/Golgi SNARE proteins for fusion with ER-derived vesicles. In contrast, the ERGIC is distinct from the ER in containing COPI and the ARFGEFs necessary to initiate COPI assembly and drive retrograde transport back to the ER. Therefore in a simplified model, the first heterotypic fusion event for COPII vesicles may be with the Golgi-like ERGIC in animal cells and the ERGIC-like cis-Golgi in yeast. Depending on the variety of secretory cargo transported in a given cell type, enlarged COPII carriers may be generated to accommodate larger cargo (Stagg et al. 2006) for transport to the ERGIC.

What is the relationship between the ERGIC and the cis-Golgi compartments? Here essentially two models have been put forward (Appenzeller-Herzog and Hauri 2006). First, retrograde-directed COPI coated vesicles could continue to bud from the ERGIC as this carrier matures and migrates toward the Golgi stack in a microtubule dependent manner. Ultimately the maturing ERGIC would then replace the cismost Golgi cisternae. This process would be consistent with a general cisternal progression model in which the ERGIC is an extension of the continually maturing Golgi stack (Glick and Luini 2011). In yeast, a parallel process could operate as the initial ERGIC-like cis-Golgi matures to acquire later Golgi markers as observed in live imaging experiments (Losev et al. 2006; Matsuura-Tokita et al. 2006). A second model describes the ERGIC as a longerlived compartment that receives COPII vesicles and then generates anterograde cargo carriers from peripheral elements of the ERGIC. Distinct functional domains within the ERGIC could be generated through segregation of recycling components into COPI rich regions with nascent cargo partitioned into peripheral elements that detach from the ERGIC. This model is consistent with in vivo observations showing that ERGIC proteins (e.g., ERGIC-53) display a steady-state localization pattern that remains adjacent to ER export sites whereas nascent secretory cargo migrates in pleiomorphic carriers that move from the ERGIC to the cis-Golgi region (Ben-Tekaya et al. 2005). However a limitation of this model is that the mechanism by which anterograde carriers may be detached from the ERGIC has not been described. It also seems possible that some combination of these models could operate in cells with a maturation pathway necessary for biogenesis of the ERGIC and Golgi compartments but once established migration of peripheral element or tubular connections could transport nascent secretory cargo.

In summary, excellent progress has been made in defining molecular mechanisms underlying membrane tethering and fusion through the use of refined cell free assays, although there is a lack of clarity in placing these reactions in specific locations and order within live cells. We hypothesize that a heterotypic fusion event occurs between COPII vesicles and the ERGIC that depends on much of the tethering and fusion machinery described in this review. However, it is not clear if this machinery is also used in multiple heterotypic and homotypic fusion events. Therefore, key questions remain regarding the organization and coordination of fusion events in the early secretory pathway. For example, how significant are homotypic fusion events in generation of the ERGIC? What are the major heterotypic fusion events that ultimately expose lumenal secretory cargo to Golgi specific processing enzymes? And at a higher level of organization what are the regulatory mechanisms that coordinate rates of fusion and vesicle budding to maintain the observed compartmental structure and function? Answers to these questions will require creative experimentation to manipulate more complex reactions in vitro as well as methods to monitor these events with greater spatial and temporal resolution in living cells.

\section{REFERENCES}

Aguilera-Romero A, Kaminska J, Spang A, Riezman H, Muñiz M. 2008. The yeast p24 complex is required for the formation of COPI retrograde transport vesicles from the Golgi apparatus. J Cell Biol 180: 713-720. 
Allan BB, Moyer BD, Balch WE. 2000. Rab1 recruitment of p115 into a cis-SNARE complex: programming budding COPII vesicles for fusion. Science 289: 444-448.

Alvarez C, Fujita H, Hubbard A, Sztul E. 1999. ER to Golgi transport: Requirement for $\mathrm{p} 115$ at a preGolgi VTC stage. J Cell Biol 147: 1205-1222.

Alvarez C, Garcia-Mata R, Hauri HP, Sztul E. 2001. The p115-interactive proteins GM130 and giantin participate in endoplasmic reticulum-Golgi traffic. J Biol Chem 276: 2693-26700.

An Y, Chen CY, Moyer B, Rotkiewicz P, Elsliger MA, Godzik A, Wilson IA, Balch WE. 2009. Structural and functional analysis of the globular head domain of $\mathrm{p} 115$ provides insight into membrane tethering. J Mol Biol 391: 26-41.

Appenzeller-Herzog C, Hauri HP. 2006. The ER-Golgi intermediate compartment (ERGIC): in search of its identity and function. J. Cell Sci 119: 2173-2183.

Baker D, Hicke L, Rexach M, Schleyer M, Schekman R. 1988. Reconstitution of SEC gene product-dependent intercompartmental protein transport. Cell 54: 335-344.

Bannykh SI, Rowe T, Balch WE. 1996. The organization of endoplasmic reticulum export complexes. J Cell Biol 135: 19-35.

Barlowe C. 1997. Coupled ER to Golgi transport reconstituted with purified cytosolic proteins. J Cell Biol 139: 1097-1108.

Barr FA. 1999. A novel Rab6-interacting domain defines a family of Golgi-targeted coiled-coil proteins. Curr Biol 9: 381-384.

Barr FA, Short B. 2003. Golgins in the structure and dynamics of the Golgi apparatus. Curr Opin Cell Biol 15: 405-413.

Barr FA, Nakamura N, Warren G. 1998. Mapping the interaction between GRASP65 and GM130, components of a protein complex involved in the stacking of Golgi cisternae. EMBO J 17: 3258-3268.

Barrowman J, Sacher M, Ferro-Novick S. 2000. TRAPP stably associates with the Golgi and is required for vesicle docking. EMBO J 19: 862-869.

Beard M, Satoh A, Shorter J, Warren G. 2005. A cryptic Rabl-binding site in the p115 tethering protein. J Biol Chem 280: 25840-25848.

Beckers CJ, Keller DS, Balch WE. 1987. Semi-intact cells permeable to macromolecules: use in reconstitution of protein transport from the endoplasmic reticulum to the Golgi complex. Cell 50: 523-534.

Behnia R, Barr FA, Flanagan JJ, Barlowe C, Munro S. 2007. The yeast orthologue of GRASP 65 forms a complex with a coiled-coil protein that contributes to ER to Golgi traffic. J Cell Biol 176: 255-261.

Belden WJ, Barlowe C. 1996. Erv25p, a component of COPII-coated vesicles, forms a complex with Emp24p that is required for efficient endoplasmic reticulum to Golgi transport. J Biol Chem 271: 26939-26946.

Ben-Tekaya H, Miura K, Pepperkok R, Hauri HP. 2005. Live imaging of bidirectional traffic from the ERGIC. J Cell Sci 118: $357-367$.

Bock JB, Matern HT, Peden AA, Scheller RH. 2001. A genomic perspective on membrane compartment organization. Nature 409: 839-841.
Bremser M, Nickel W, Schweikert M, Ravazzola M, Amherdt M, Hughes CA, Söllner TH, Rothman JE, Wieland FT. 1999. Coupling of coat assembly and vesicle budding to packaging of putative cargo receptors. Cell 96: 495-506.

Bui QT, Golinelli-Cohen MP, Jackson CL. 2009. Large Arf1 guanine nucleotide exchange factors: Evolution, domain structure, and roles in membrane trafficking and human disease. Mol Genet Genomics 282: 329-350.

Cai H, Reinisch K, Ferro-Novick S. 2007a. Coats, tethers, Rabs, and SNAREs work together to mediate the intracellular destination of a transport vesicle. Dev Cell 12: 671-82.

Cai Y, Chin HF, Lazarova D, Menon S, Fu C, Cai H, Sclafani A, Rodgers DW, De La Cruz EM, Ferro-Novick S, et al. 2008. The structural basis for activation of the Rab Yptlp by the TRAPP membrane-tethering complexes. Cell 133: 1202-1213.

Cai H, Yu S, Menon S, Cai Y, Lazarova D, Fu C, Reinisch K, Hay JC, Ferro-Novick S. 2007b. TRAPPI tethers COPII vesicles by binding the coat subunit Sec23. Nature 445: 941-944.

Cao X, Ballew N, Barlowe C. 1998. Initial docking of ER-derived vesicles requires Usolp and Yptlp but is independent of SNARE proteins. EMBO J 17: 21562165.

Dascher C, Balch WE. 1996. Mammalian Sly1 regulates syntaxin 5 function in endoplasmic reticulum to Golgi transport. J Biol Chem 271: 15866-15869.

Dascher C, Ossig R, Gallwitz D, Schmitt HD. 1991. Identification and structure of four yeast genes (SLY) that are able to suppress the functional loss of YPT1, a member of the RAS superfamily. Mol Cell Biol 11: 872-885.

Dulubova I, Khvotchev M, Liu S, Huryeva I, Südhof TC, Rizo J. 2007. Munc18-1 binds directly to the neuronal SNARE complex. Proc Natl Acad Sci 104: 2697-2702.

Fasshauer D, Sutton RB, Brunger AT, Jahn R. 1998. Conserved structural features of the synaptic fusion complex: SNARE proteins reclassified as Q- and R-SNAREs. Proc Natl Acad Sci 95: 15781-15786.

Furgason ML, MacDonald C, Shanks SG, Ryder SP, Bryant NJ, Munson M. 2009. The N-terminal peptide of the syntaxin Tlg2p modulates binding of its closed conformation to Vps45p. Proc Natl Acad Sci 106: 14303-14308.

García-Mata R, Szul T, Alvarez C, Sztul E. 2003. ADP-ribosylation factor/COPI-dependent events at the endoplasmic reticulum-Golgi interface are regulated by the guanine nucleotide exchange factor GBF1. Mol Biol Cell 14: $2250-2261$.

Gillingham AK, Pfeifer AC, Munro S. 2002. CASP, the alternatively spliced product of the gene encoding the CCAAT-displacement protein transcription factor, is a Golgi membrane protein related to giantin. Mol Biol Cell 13: 3761-3774.

Gillingham AK, Tong AH, Boone C, Munro S. 2004. The GTPase Arflp and the ER to Golgi cargo receptor Erv14p cooperate to recruit the golgin Rud3p to the cisGolgi. J Cell Biol 167: 281-292.

Glick BS, Luini A. 2011. Models for Golgi traffic: a critical assessment. Cold Spring Harb Perspect Biol doi:10.1101/ cshperspect.a005215. 
Hay JC, Chao DS, Kuo CS, Scheller RH. 1997. Protein interactions regulating vesicle transport between the endoplasmic reticulum and Golgi apparatus in mammalian cells. Cell 89: 149-158.

Hay JC, Klumperman J, Oorschot V, Steegmaier M, Kuo CS, Scheller RH. 1998. Localization, dynamics, and protein interactions reveal distinct roles for ER and Golgi SNAREs. J Cell Biol 141: 1489-502.

Jang SB, Kim YG, Cho YS, Suh PG, Kim KH, Oh BH. 2002. Crystal structure of SEDL and its implications for a genetic disease spondyloepiphyseal dysplasia tarda. $J$ Biol Chem 277: 49863-49869.

Jiang Y, Scarpa A, Zhang L, Stone S, Feliciano E, FerroNovick S. 1998. A high copy suppressor screen reveals genetic interactions between BET3 and a new gene. Evidence for a novel complex in ER-to-Golgi transport. Genetics 149: 833-841.

Jones S, Newman C, Liu F, Segev N. 2000. The TRAPP complex is a nucleotide exchanger for Ypt1 and Ypt31/32. Mol Biol Cell 11: 4403-4411.

Kim YG, Raunser S, Munger C, Wagner J, Song YL, Cygler M, Walz T, Oh BH, Sacher M. 2006. The architecture of the multisubunit TRAPP I complex suggests a model for vesicle tethering. Cell 127: 817-830.

Kondylis V, Rabouille C. 2003. A novel role for dp115 in the organization of tER sites in Drosophila. J Cell Biol 162: $185-198$.

Kondylis V, Spoorendonk KM, Rabouille C. 2005. dGRASP localization and function in the early exocytic pathway in Drosophila S2 cells. Mol Biol Cell 16: 4061-4072.

Lee MC, Miller EA, Goldberg J, Orci L, Schekman R. 2004. Bi-directional protein transport between the ER and Golgi. Annu Rev Cell Dev Biol 20: 87-123.

Letourneur F, Gaynor EC, Hennecke S, Démollière C, Duden R, Emr SD, Riezman H, Cosson P. 1994. Coatomer is essential for retrieval of dilysine-tagged proteins to the endoplasmic reticulum. Cell 79: 1199-1207.

Levi SK, Bhattacharyya D, Strack RL, Austin JR II, Glick BS 2010. The yeast GRASP Grh1 colocalizes with COPII and is dispensable for organizing the secretory pathway. Traffic 11: 1168-1179.

Lippincott-Schwartz J, Yuan LC, Bonifacino JS, Klausner RD. 1989. Rapid redistribution of Golgi proteins into the ER in cells treated with brefeldin A: Evidence for membrane cycling from Golgi to ER. Cell 56: 801-813.

Losev E, Reinke CA, Jellen J, Strongin DE, Bevis BJ, Glick BS. 2006. Golgi maturation visualized in living yeast. Nature 441: $1002-1006$

Malhotra V, Serafini T, Orci L, Shepherd JC, Rothman JE. 1989. Purification of a novel class of coated vesicles mediating biosynthetic protein transport through the Golgi stack. Cell 58: 329-336.

Matsuura-Tokita K, Takeuchi M, Ichihara A, Mikuriya K, Nakano A. 2006. Live imaging of yeast Golgi cisternal maturation. Nature 441: 1007-1010.

Mogelsvang S, Gomez-Ospina N, Soderholm J, Glick BS, Staehelin LA. 2003. Tomographic evidence for continuous turnover of Golgi cisternae in Pichia pastoris. Mol Biol Cell 14: 2277-2291.
Morin-Ganet MN, Rambourg A, Deitz SB, Franzusoff A, Képès F. 2000. Morphogenesis and dynamics of the yeast Golgi apparatus. Traffic 1: 56-68.

Morohashi Y, Balklava Z, Ball M, Hughes H, Lowe M. 2010. Phosphorylation and membrane dissociation of the ARF exchange factor GBF1 in mitosis. Biochem J 427: 401-412.

Mouratou B, Biou V, Joubert A, Cohen J, Shields DJ, Geldner N, Jürgens G, Melançon P, Cherfils J. 2005. The domain architecture of large guanine nucleotide exchange factors for the small GTP-binding protein Arf. BMC Genomics 6: 20 .

Moyer BD, Allan BB, Balch WE. 2001. Rab1 interaction with a GM130 effector complex regulates COPII vesicle cisGolgi tethering. Traffic 2: 268-276.

Munro S. 2011. The Golgin Coiled-Coil Proteins of the Golgi Apparatus. Cold Spring Harb Perspect Biol doi:10.1101/cshperspect.a005256.

Nakajima H, Hirata A, Ogawa Y, Yonehara T, Yoda K, Yamasaki M. 1991. A cytoskeleton-related gene, usol, is required for intracellular protein transport in Saccharomyces cerevisiae. J Cell Biol 113: 245-260.

Niu TK, Pfeifer AC, Lippincott-Schwartz J, Jackson CL. 2005. Dynamics of GBF1, a Brefeldin A-sensitive Arf1 exchange factor at the Golgi. Mol Biol Cell 16: 1213-1222.

Novick P, Field C, Schekman R. 1980. Identification of 23 complementation groups required for post-translational events in the yeast secretory pathway. Cell 21: 205-215.

Ossig R, Dascher C, Trepte HH, Schmitt HD, Gallwitz D. 1991. The yeast SLY gene products, suppressors of defects in the essential GTP-binding Yptl protein, may act in endoplasmic reticulum-to-Golgi transport. Mol Cell Biol 11: 2980-2993.

Ostermann J, Orci L, Tani K, Amherdt M, Ravazzola M, Elazar Z, Rothman JE. 1993. Stepwise assembly of functionally active transport vesicles. Cell 75: 1015-1025.

Palade G. 1975. Intracellular aspects of the process of protein synthesis. Science 189: 347-358.

Parlati F, McNew JA, Fukuda R, Miller R, Söllner TH, Rothman JE. 2000. Topological restriction of SNAREdependent membrane fusion. Nature 407: 194-198.

Pavel J, Harter C, Wieland FT. 1998. Reversible dissociation of coatomer: functional characterization of a $\beta / \delta$-coat protein subcomplex. Proc Natl Acad Sci 95: 2140-2145.

Peng R, Gallwitz D. 2002. Sly1 protein bound to Golgi syntaxin Sed5p allows assembly and contributes to specificity of SNARE fusion complexes. J Cell Biol 157: 645-655.

Pepperkok R, Scheel J, Horstmann H, Hauri HP, Griffiths G, Kreis TE. 1993. $\beta$-COP is essential for biosynthetic membrane transport from the endoplasmic reticulum to the Golgi complex in vivo. Cell 74: 71-82.

Peyroche A, Paris S, Jackson CL. 1996. Nucleotide exchange on ARF mediated by yeast Geal protein. Nature 384: 479-481.

Peyroche A, Antonny B, Robineau S, Acker J, Cherfils J, Jackson CL. 1999. Brefeldin A acts to stabilize an abortive ARF-GDP-Sec7 domain protein complex: involvement of specific residues of the Sec7 domain. Mol Cell 3: $275-285$. 
Presley JF, Cole NB, Schroer TA, Hirschberg K, Zaal KJ, Lippincott-Schwartz J. 1997. ER-to-Golgi transport visualized in living cells. Nature 389: 81-85.

Puthenveedu MA, Bachert C, Puri S, Lanni F, Linstedt AD. 2006. GM130 and GRASP65-dependent lateral cisternal fusion allows uniform Golgi-enzyme distribution. Nat Cell Biol 8: 238-248.

Puthenveedu MA, Linstedt AD. 2001. Evidence that Golgi structure depends on a p115 activity that is independent of the vesicle tether components giantin and GM130. $J$ Cell Biol 155: 227-238.

Puthenveedu MA, Linstedt AD. 2004. Gene replacement reveals that $\mathrm{p} 115 /$ SNARE interactions are essential for Golgi biogenesis.Proc Natl Acad Sci 101: 1253-1256.

Rambourg A, Clermont Y, Jackson CL, Képès F. 1994. Ultrastructural modifications of vesicular and Golgi elements in the Saccharomyces cerevisiae sec 21 mutant at permissive and non-permissive temperatures. Anat 240: 32-41.

Rein U, Andag U, Duden R, Schmitt HD, Spang A. 2002. ARF-GAP-mediated interaction between the ER-Golgi v-SNAREs and the COPI coat. J. Cell Biol 157: 395-404.

Rossi G, Kolstad K, Stone S, Palluault F, Ferro-Novick S. 1995. BET3 encodes a novel hydrophilic protein that acts in conjunction with yeast SNAREs. Mol Biol Cell 6: 1769-1780.

Rothman JE. 1994. Mechanisms of intracellular protein transport. Nature 372: 55-63.

Rowe T, Dascher C, Bannykh S, Plutner H, Balch WE. 1998. Role of vesicle-associated syntaxin 5 in the assembly of pre-Golgi intermediates. Science 279: 696-700.

Sacher M, Barrowman J, Wang W, Horecka J, Zhang Y, Pypaert M, Ferro-Novick S. 2001. TRAPP I implicated in the specificity of tethering in ER-to-Golgi transport. Mol Cell 7: 433-442.

Sacher M, Jiang Y, Barrowman J, Scarpa A, Burston J, Zhang L, Schieltz D, Yates JR III, Abeliovich H, Ferro-Novick S. 1998. TRAPP, a highly conserved novel complex on the cis-Golgi that mediates vesicle docking and fusion. EMBO J 17: 2494-2503.

Sapperstein SK, Lupashin VV, Schmitt HD, Waters MG. 1996. Assembly of the ER to Golgi SNARE complex requires Uso1p. J Cell Biol 132: 755-767.

Sapperstein SK, Walter DM, Grosvenor AR, Heuser JE, Waters MG. 1995. p115 is a general vesicular transport factor related to the yeast endoplasmic reticulum to Golgi transport factor Usolp. Proc Natl Acad Sci 92: 522-526.

Scales SJ, Pepperkok R, Kreis TE. 1997. Visualization of ER-to-Golgi transport in living cells reveals a sequential mode of action for COPII and COPI. Cell 90: 1137-1148.

Schekman R, Orci L. 1996. Coat proteins and vesicle budding. Science 271: 1526-1533.

Serafini T, Orci L, Amherdt M, Brunner M, Kahn RA, Rothman JE. 1991. ADP-ribosylation factor is a subunit of the coat of Golgi-derived COP-coated vesicles: a novel role for a GTP-binding protein. Cell 67: 239-253.

Shen J, Tareste DC, Paumet F, Rothman JE, Melia TJ. 2007. Selective activation of cognate SNAREpins by Secl/ Munc18 proteins. Cell 128: 183-195.
Shima DT, Scales SJ, Kreis TE, Pepperkok R. 1999. Segregation of COPI-rich and anterograde-cargo-rich domains in endoplasmic-reticulum-to-Golgi transport complexes. Curr Biol 9: 821-824.

Shorter J, Beard MB, Seemann J, Dirac-Svejstrup AB, Warren G. 2002. Sequential tethering of Golgins and catalysis of SNAREpin assembly by the vesicle-tethering protein p115. J Cell Biol 157: 45-62.

Malsam J, Söllner TH. 2011.Organization Organization of SNAREs within the Golgi stack. Cold Spring Harb Perspect Biol doi:10.1101/cshperspect.a005249.

Sönnichsen B, Lowe M, Levine T, Jamsa E, Dirac-Svejstrup B, Warren G. 1998. A role for giantin in docking COPI vesicles to Golgi membranes. J Cell Biol 140: 1013-1021.

Spang A, Schekman R. 1998. Reconstitution of retrograde transport from the Golgi to the ER in vitro. J Cell Biol 143: $589-599$.

Spang A, Shiba Y, Randazzo PA. 2010. Arf GAPs: Gatekeepers of vesicle generation. FEBS Lett 584: 2646-2651.

Springer S, Spang A, Schekman R. 1999. A primer on vesicle budding. Cell 97: 145-148.

Stagg SM, Gürkan C, Fowler DM, LaPointe P, Foss TR, Potter CS, Carragher B, Balch WE. 2006. Structure of the Sec13/31 COPII coat cage. Nature 439: 234-238.

Stamnes MA, Craighead MW, Hoe MH, Lampen N, Geromanos S, Tempst P, Rothman JE. 1995. An integral membrane component of coatomer-coated transport vesicles defines a family of proteins involved in budding. Proc Natl Acad Sci 92: 8011-8015.

Südhof TC, Rothman JE. 2009. Membrane fusion: Grappling with SNARE and SM proteins. Science 323: 474-477.

Tang D, Yuan H, Wang Y. 2010. The role of GRASP65 in Golgi cisternal stacking and cell cycle progression. Traffic 11: $827-842$.

VanRheenen SM, Cao X, Sapperstein SK, Chiang EC, Lupashin VV, Barlowe C, Waters MG. 1999. Sec34p, a protein required for vesicle tethering to the yeast Golgi apparatus, is in a complex with Sec35p. J Cell Biol 147: 729-742.

Wang W, Sacher M, Ferro-Novick S. 2000. TRAPP stimulates guanine nucleotide exchange on Ypt1p. J Cell Biol 151: 289-296.

Ward TH, Polishchuk RS, Caplan S, Hirschberg K, Lippincott-Schwartz J. 2001. Maintenance of Golgi structure and function depends on the integrity of ER export. $J$ Cell Biol 155: 557-570.

Waters MG, Serafini T, Rothman JE. 1991. 'Coatomer': A cytosolic protein complex containing subunits of non-clathrin-coated Golgi transport vesicles. Nature 349: 248-251.

Weber T, Zemelman BV, McNew JA, Westermann B, Gmachl M, Parlati F, Söllner TH, Rothman JE. 1998. SNAREpins: minimal machinery for membrane fusion. Cell 92: $759-772$.

Weide T, Bayer M, Köster M, Siebrasse JP, Peters R, Barnekow A. 2001. The Golgi matrix protein GM130: a specific interacting partner of the small GTPase rablb. EMBO Rep 2001 2: 336-341. 
Entry/Exit from the cis-Face of the Golgi Complex

Wieland FT. 2011. COPI Budding within the Golgi Stack. Cold Spring Harb Perspect Biol doi:10.1101/ cshperspect.a005231.

Xu D, Hay JC. 2004. Reconstitution of COPII vesicle fusion to generate a pre-Golgi intermediate compartment. J Cell Biol 167: 997-1003.

Xu D, Joglekar AP, Williams AL, Hay JC. 2000. Subunit structure of a mammalian ER/Golgi SNARE complex. J Biol Chem 275: 39631-39639.

Yamakawa H, Seog DH, Yoda K, Yamasaki M, Wakabayashi T. 1996. Usol protein is a dimer with two globular heads and a long coiled-coil tail. J Struct Biol 116: 356-365.
Yu I-M, Hughson FM. 2010. Tethering factors as organizers of intracellular vesicular traffic. Ann Rev Cell Dev Biol 26: 137-156.

Yu S, Satoh A, Pypaert M, Mullen K, Hay JC, Ferro-Novick S. 2006. mBet3p is required for homotypic COPII vesicle tethering in mammalian cells. J Cell Biol 174: 359-368.

Zhao X, Lasell TK, Melançon P. 2002. Localization of large ADP-ribosylation factor-guanine nucleotide exchange factors to different Golgi compartments: evidence for distinct functions in protein traffic. Mol Biol Cell 13: 119-133. 


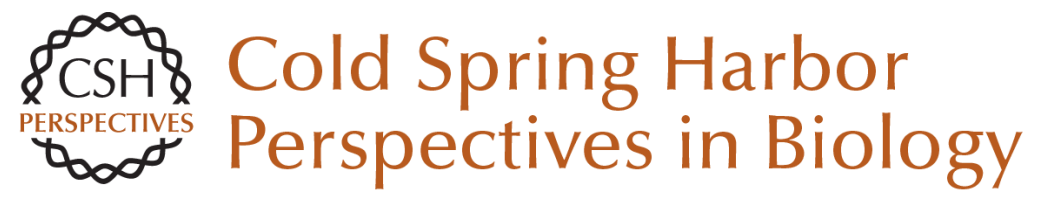

\section{Entry and Exit Mechanisms at the cis-Face of the Golgi Complex}

Andrés Lorente-Rodríguez and Charles Barlowe

Cold Spring Harb Perspect Biol 2011; doi: 10.1101/cshperspect.a005207 originally published online April 11,2011

\section{Subject Collection The Golgi}

Structure of Golgi Transport Proteins

Daniel Kümmel and Karin M. Reinisch

\section{Golgi Biogenesis}

Yanzhuang Wang and Joachim Seemann

Golgi Glycosylation and Human Inherited

Diseases

Hudson H. Freeze and Bobby G. Ng

Models for Golgi Traffic: A Critical Assessment Benjamin S. Glick and Alberto Luini

\section{Architecture of the Mammalian Golgi} Judith Klumperman

Evolution and Diversity of the Golgi Mary J. Klute, Paul Melançon and Joel B. Dacks

Evolutionary Forces Shaping the Golgi

Glycosylation Machinery: Why Cell Surface

Glycans Are Universal to Living Cells Ajit Varki

Golgi Positioning

Smita Yadav and Adam D. Linstedt
Golgi and Related Vesicle Proteomics: Simplify to Identify Joan Gannon, John J.M. Bergeron and Tommy Nilsson

Organization of SNAREs within the Golgi Stack Jörg Malsam and Thomas H. Söllner

Golgi during Development Weimin Zhong

Entry and Exit Mechanisms at the cis-Face of the Golgi Complex Andrés Lorente-Rodríguez and Charles Barlowe

COPI Budding within the Golgi Stack Vincent Popoff, Frank Adolf, Britta Brügger, et al.

Mechanisms of Protein Retention in the Golgi David K. Banfield

The Golgin Coiled-Coil Proteins of the Golgi

Apparatus Sean Munro

Signaling at the Golgi Peter Mayinger

For additional articles in this collection, see http://cshperspectives.cshlp.org/cgi/collection/

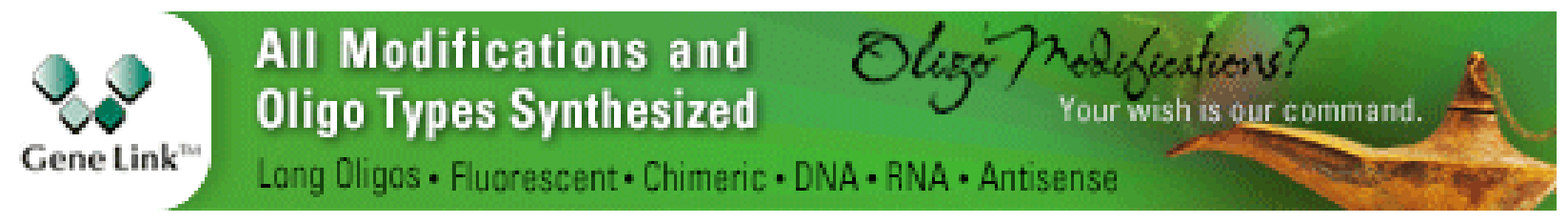

Copyright @ 2011 Cold Spring Harbor Laboratory Press; all rights reserved 